\title{
LA NOZIONE DI ALTRO NELLA TEORIA KIERKEGAARDIANA DEGLI STADI ESISTENZIALI
}

\author{
João Marcelo Crubellate ${ }^{1}$
}

\begin{abstract}
SOMMARIO: Partendo dalla nozione kierkegaardiana dell'Io come un rapportarsi propongo discutere in questo testo il concetto di altro nei diversi stadi esistenziali: l'estetico, l'etico ed il religioso. Cerco prima di descrivire il problema dell'Io nella filosofia di Kierkegaard rispetto alla questione da lui enunciata fin dall'inizio del saggio sulla Malattia Mortale (KIERKEGAARD, 2013a), ossia che la coscienza possa porre a se stesso o debba esser posta da un altro. Poi la mia ricerca appunta verso la nozione dell'altro nell'estetico come occasione per il godere del se stesso e appunta anche verso la nozione dell'altro come immagine dell' $I o$, un secondo $I o$, nello stadio etico. Alla fine arrivo al religioso dove, per il teologo danese, l'altro prende il senso di un altro come altro, una realtà in sé, dato che nel religioso l'altro è, prima di tutto, il Dio - la vera fonte della realtà dell' Io stesso - ed è ancora il prossimo, tutte le altre persone che, insieme all' $I o$, devono nella sua singolarità diventare spirito, coscienza coesa nella esistenza. Quest'altro religioso è pertanto il punto centrale della filosofia sociale kierkegaardiana, giacché nella posizione del prossimo egli è infatti il primo Tu (KIERKEGAARD, 2003).
\end{abstract}

PAROLE CHIAVI: Kierkegaard. Rapportarsi. Stadio esistenziale. Prossimo.

\section{INTRODUZIONE}

In questo articolo io propongo discutere la nozione kierkegaardiana di essere umano come un essere relazionale, ciò che appunta ad una filosofia sociale nell'ampio compito del pensiero del solitario danese. A questo proposito aggiungo un altro: ricercare il senso che la nozione di altro prende in ogni stadio esistenziale kierkegaardiano, cioè l'estetico, l'etico e alla fine il religioso.

Per raggiungere questi propositi dovrò discutere un punto centrale della filosofia kierkegaardiana, vale a dire quel problema del fondamento del

\footnotetext{
${ }^{1}$ Professor na Universidade Estadual de Maringá e doutorando no Programa de Pós-Graduação em Filosofia da Pontifícia Universidade Católica do Paraná - PUC/PR, com estágio concluído na Pontifícia Università Gregoriana, em Roma. E-mail: jmcrubellate@uem.br
} 
sé come una soggettività nel tempo, nella esistenza, una singularità che diventa così in libertà ovvero come prodotto delle scelte che l'uomo stesso fa ogni istante della sua vita, verso ad uno scopo o una meta che, pure questo, lui accoglie o rigetta (ma non ne può creare liberamente). Su questo la filosofia di Kierkegaard appare come paradossale, dato che la discussione della nozione di altro non può esser fatta senza che sia fatta insieme e allo stesso tempo la discussione sul processo per cui l'uomo diventa singularità. Ciò che possiamo chiamare l'Io prende il suo senso, nel pensiero di Kierkegaard, soltanto nel processo del rapportarsi ossia nell'ambito della soggettività, perché è rapporto, in primo luogo, con se stesso, e nell'ambito della sociabilità, perché è anche rapporto con gli altri, con Dio e con gli altri uomini.

A questo fondamento del sé nella esistenza si deve aggiungere un altro, quello della libertà come condizione dell'attuarsi umano verso a se stesso la scelta di se stesso (KIERKEGAARD, 1980, p. 49) - e dunque verso alle altre persone. La premessa fondante della risposta kierkegaardiana al problema della libertà del sé, vale dire la premessa dell'antropologia kierkegaardiana, è la affermazione dell'uomo come un rapporto che si rapporta a se stesso ossia una apertura ed una possibilità, possibilità di divenire, un progetto sebbene non ancora un Io coerente con se stesso (KIERKEGAARD, 2008, p. 17). Il movimento dalla possibilità alla realtà esistenziale dell' $I o$, la costituzione del suo fondamento ontologico - il porre a se stesso nell'esistenza (KIERKEGAARD, 2013a, p. 1665; LÜBCKE, 2008, p. 73-88) - segue lo scopo che l'uomo stesso accoglie per sé.

Ma anche il porre a se stesso comprende più di una possibilità, dal momento che "un tale rapporto che si rapporta a se stesso, un sé, deve o aver determinato esso stesso il fine delle proprie azioni e della propria vita, oppure trarre la fondazione di queste norme da qualcos'altro" (LÜBCKE, 2008, p. 84). Nella sua libertà e proprio a causa della sua libertà l'uomo può fare le scelte che lo conducono a esser determinato da un altro ossia esternamente. Però ci si può ancora sospettare che la facoltà di porre a se stesso non sia una reale possibilità per un essere finito, o che non sia una facoltà che si svolga nell'isolamento del sé.

Il problema si sviluppa intorno a più di una soluzione, come cerco di dimostrare nella continuazione di questo testo. Poi vedremo che il rapporto del sé verso all'altro prende una molteplicità di sensi secondo lo stadio esistenziale analizzato e che questa molteplicità è importante anche per l'analisi della filosofia sociale kierkegaardiana, specialmente sviluppata sotto la nozione di prossimo, 
il concetto specifico con cui Kierkegaard parla dell'altro umano, nel stadio religioso. Questo rapporto teorico fra la nozione di altro e la teoria degli stadi esistenziali è il contributo specifico che voglio offrire con questa discussione.

Alla fine del testo spero che si possa raggiungere, tra molti altri punti, almeno due importanti conclusioni: l'evvidenza di una filosofia sociale kierkegaardiana, per cui la nozione dell'attuarsi del soggetto umano è fondamentale, e l'evvidenza di che è nell'ambito del religioso - con la nozione di un altro come il prossimo e la piena coscienza della incompiutezza ontologica della natura umana cioè la sua finitudine originaria - che la vita sociale prende, dal punto di vista di Kierkegaard, il suo più nobile significato ${ }^{2}$ dato che è nel religioso che l'Io diventa l'Io-insieme-all'altro.

\section{L'Io COME RAPPORTO COSTITUTIVO DEL SÉ NELLA ESISTENZA: L'Io è UN RAPPORTARSI}

Secondo alcuni ricercatori del pensiero di Kierkegaard (MORELLI, 2012, p. 136-138; FABRO, 2004, p. 272-273; REGINA, 2008, p. 1214; PIAZZESI, 2009, p. 145-161), il concetto di rapporto è importante per discutere le nozioni di soggettività, verità ed esistenza, i nodi concettuali decisivi nel pensiero del solitario danese. $\mathrm{Ci}$ sono almeno tre possibili direzioni per discutere l'affermazione di Kierkegaard rispetto alla definizione di uomo come rapporto - il punto di partenza per una filosofia sociale kierkegaardiana - vale a dire che "[...] l'uomo [...] è un rapportarsi [...] e [...] un tale rapporto che si rapporta a se stesso, un io, o deve essere posto da sé o deve essere stato posto da un altro" (KIERKEGAARD, 2008, p. 17). La prima è quella che fa dell'altro il fondamento della relazione fra l'individuo come un sé e l'altro. Poi la direzione che fa della relazione del sé con se stesso il fondamento. E alla fine quella direzione che voglio proporre in questo testo e nella quale il sé e l'altro di sé sono messi insieme come fondamenti del rapportarsi costitutivo dell'umano ${ }^{3}$.

Quello che si può intendere con questa terza possibilità è stabilire un'alternativa di analisi per il problema del rapportarsi che sia allo stesso tempo esistenziale e ontologicamente orientata. Per questa ragione è che il concetto

\footnotetext{
${ }^{2}$ Anche se questa dimensione sociale sia già palese nella così detta etica-seconda (ALMEIDA, 2009; KIERKEGAARD, 1992), cioè nel diventare se stesso, la mia è una prospettiva che cerca di puntare verso l'altro come punto fondamentale di quello processo e, per questo, il religioso - come espresso nel saggio sugli Atti dell'amore - sarà il mio punto focale.

${ }^{3}$ Quest'è la prospettiva del religioso (per esempio FABRO, 2014, p. 5-6).
} 
più importante - anche più che i concetti del sé e di altro - è quello di rapporto: l'umano per Kierkegaard è, insomma, un rapporto, ma un rapporto che si rapporta a se stesso (KIERKEGAARD, 2013a, p. 1671; GRØN, 2008, p. 105).

La questione dell'Io è così centrale nel pensiero di Kierkegaard che a volta diventa un punto di attacco per che lui sia giudicato come un pensatore soggettivista ed anche solipsistico. Ma questa non è certamente una buona interpretazione del concetto nella filosofia kierkegaardiana perché, come si vedrà, l'Io non è un ente che si diventi da solo, che si possa aggiornare o sia posto nella esistenza senza il concorso di un altro (secondo Kierkegaard l'uomo è sintesi - ambivalenza costituttiva - tra singolo e società, come scrive FABRO, 2010, p. 21). Che ci sia nella filosofia kierkegaardiana una vera ambivalenza e pure un'esteriorità fra l'Io e l'altro come i punti di una vera dialettica è anche un aspetto a non esser dimenticato. L'inizio del saggio sulla Malattia Mortale è così importante per questo dibattito e così difficile di essere compreso che merita di esser trascrito:

Luomo è spirito. Ma cos’è lo spirito? Lo spirito è l'io. E l'io cos’è? È un rapporto che si rapporta a se stesso oppure è, nel rapporto, il rapportarsi che il rapporto si rapporta a se stesso; l'io non è il rapporto, ma il rapportarsi a se stesso. L'uomo è una sintesi ... Un tale rapporto che si rapporta a se stesso, un io, o deve essere posto da se stesso o deve essere stato posto da un altro. [...] Un tale rapporto derivato, posto, è l'io dell'uomo; un rapporto che si mette in rapporto con se stesso e, mettendosi in rapporto con se stesso, si mette in rapporto con un altro (KIERKEGAARD, 2013a, p. 1665).

$\mathrm{Ci}$ sono molti punti fondamentali in questo brano ma il nocciolo dell'intero testo è il rapportarsi, l'azione del rapporto che si rapporta a se stesso come il processo per cui l'uomo si può attuare. Quello che Kierkegaard afferma è che l'Io non è il rapporto tra finito e infinito, tempo e eternità, ecc, ma è il rapportarsi del rapporto tra finito e infinito, tempo e eternità, ecc, con se stesso. L'Io è il rapportarsi di un rapporto con se stesso. Ma cos'è questo? L'uomo è una relazione, sintesi tra finito e infinito, tempo e eternità, ecc. Ma non é ancora un $I o$, uno spirito (coscienza). L'Io è il rapportarsi del rapporto con se stesso, ciò che significa dire che l'Io è il rapporto cosciente tra finito e infinito, tempo e eternità nell'uomo ${ }^{4}$. L'Io è perciò il percorso della coscienza (un dialogo interiore) verso a se stessa (o anche è l'autocoscienza), verso all'integrazione del sé con se stesso, l'integrità e coesione, posta da se stesso o

${ }^{4} \mathrm{O}$ come scrive De Paula (2009, p. 112), una relazione positiva che così si riconosce. 
da un altro, che è la questione esternata da Kierkegaard alla fine della difficile mezza-pagina iniziale del suo saggio. Insomma, se l'uomo è un rapporto tra principi diversi, l'Io è in primo luogo la coscienza di quelli principi e poi è anche un percorso verso la cosciente determinazione di quello rapporto per dare alla propria esistenza un senso al di là della natura e del dissiparsi del sé nelle possibilità occasionali offerti in ogni momento della esistenza.

Questo rapportarsi fondamentale per il divenire umano è il primo punto che io vorrei prendere del branno sopraccitato ed il secondo è questo: che il rapportarsi a se stesso - l'azione o pure l'atteggiamento verso a se stesso - è posto dal proprio Io o deve esser stato posto da un altro. Quest'aparente antinomia fondamentale compresa nel porre l'Io nel mondo (" [...] o deve essere posto da se stesso o deve essere stato posto da un altro") dovrebbe esser presa come un vero eitherlor, come un'oposizione di contrari, una compresenza ativa di contrari che così appartiene allo spirito (FABRO, 2010, p. 19), nel senso kierkegaardiano di un paradosso.

In questo senso paradossale per cui arriviamo al terzo punto staccato dal brano sopracitato, nonostante che siano esterni e contrari fra di loro il porre a se stesso ed il esser stato posto da un altro, la possibilità di rapportarsi a se stesso (propriamente l'Io, lo spirito) è un rapporto all'altro quando diventa veramente rapporto con se stesso ed è, come io aggiungerò, un rapporto a se stesso quando diventa veramente rapporto ad un altro.

Secondo Lübcke (2008, p. 73-74) la discussione intorno al sé dell'uomo prende il suo senso dal fatto che l'uomo sia un campo di possibilità, mentre la natura sia sottoposta in tutto e per tutto alla necessità. La differenza decorre dal punto che solo l'uomo si rapporti a se stesso, processo per cui l'esistenza diventa per lui temporalità ossia una seguenza di momenti sensa coerenza necessaria. In questa temporalità l'uomo affronta l'esigenza di scelta e di porre a se stesso come un Io coerente, come si può vedere nell'afermazione di Lübcke (2008, p. 73-74):

L'animale vive la sua vita in una serie di punti del tempo che si susseguono in successione, mentre l'uomo si trova nell'istante, in cui 'il tempo e l'eternità si toccano'. In questo istante l'uomo, al contrario dell'animale, è posto di fronte al compito di contribuire a formare una connessione nella própria vita, nella responsabilità di fronte all'eternità. 
Quindi anche se per gli altri animali l'esistenza possa esser definitta come sintesi tra finito ed infinito - dal momento che anche un animale c'è un'essenza identitaria, una identità in relazione alla generalità di tutto il mondo rimanente e della specie a cui egli appartene (LÜBCKE, 2008, p. 73-74) tuttavia solo l'uomo può esser autoconsciente di questa infinitudine identitaria e del compito che resulta di questa condizione. Agli animali tutta conessione possibile fra i momenti della vita o continuità esistenziale è già data, determinata dal suo primo momento per la natura. All'uomo invece quella conessione deve essere la sua occupazione, il suo mestiere e di questo segue che sia anche la vera fonte della disperazione, quel "[...] vantaggio dell'uomo di fronte all'animale" (KIERKEGAARD, 2013a, p. 1667). E la disperazione decorre dalla libertà che è posta per la sintesi tra possibilità e necessità: siccome un'esistenza coerente è una possibilità ma non è una determinazione - dal momento che diventare spirito è una possibilità ma non è una determinazione - l'uomo si dispera. Però quando si dispera, comincia a lavorare per diventare spirito. Questo é il processo per cui l'uomo diventa umano, spirito, l'Io: il processo dell'attuarsi, in cui il suo lavoro, il suo compito, è il rapportarsi. Lo stesso Lübcke ci presenta al meno due interpretazione per il concetto di uomo come rapporto a se stesso: l'interpretazione esclusivamente teologica (LÜBCKE, 2008, p. 76) e l'interpretazione estetico-dogmatica (LÜBCKE, 2008, p. 81). Come si vedrà tra poco, la mia è una prospettiva che cerca di andare oltre l'esclusiva interpretazione teologica o dogmatica, dell'esclusivo essere posto o porre a se stesso.

La prima interpretazione del rapporto a se stesso conclude che, in essendo l'uomo un essere finito, la condizione perché lui possa rapportarsi a se stesso può decorrere soltanto da un Altro: da Dio. Non ci dobbiamo dimenticare che il rapportarsi a se stesso è anche la condizione ontologica dell'aggiornarsi o dell'attuarsi, del porre a se stesso o esser posto da un altro nella esistenza quotidiana. Dal punto di vista della interpretazione esclusivamente teologica il concetto kierkegaardiano non contiene il primo senso: gli autori della interpretazione esclusivamente teologica "[...] sono d'accordo nell'escludere la possibilità che il sé abbia 'posto se stesso'. In Kierkegaard in generale e in Anti-Climacus in particolare, l'uomo è 'posto da un altro', e questo altro è Dio" (LÜBCKE, 2008, p. 80). Il problema, secondo quella tradizione interpretativa, riposa sulla finitudine dell'uomo. Un essere esistente ovvero finito non può essere il fondamento ontologico per se stesso e dunque non può aver posto se stesso. Se allora l'Io deve attuarsi per mezzo del rapporto a se stesso, questo rapportarsi a se stesso prende come determinazione un altro del sé. 
In questo senso il sé è derivato, ed al testo di Kierkegaard (“[...] un tale rapporto che si rapporta a se stesso, un io, o deve essere posto da sé o deve essere stato posto da un altro" - KIERKEGAARD, 2013a, p. 1665) l'unica possibile replica è la seconda alternativa, quella di un Io posto da un altro: "[...] il sé in quanto derivato ha già sempre un'esperienza del rapporto di dipendenza" (LÜBCKE, 2008, p. 77). E quest'altro è, per dirlo ancora, il Dio. Però sapiamo che l'uomo, per Kierkegaard, non è definito come semplice finitudine, anzi come sintesi tra finito e infinito, tra tempo ed eternità. E poi quella del solitario danese non è propriamente una questione che comporti un dubbio: il tratatto della disperazione è fondato, come lui scrive già nella seconda pagina del suo testo, sulla conclusione che l'Io dell'uomo non sia posto da sé (KIERKEGAARD, 2013a, p. 1665) però "[...] si fonda [...] nella potenza che l'ha posto" (KIERKEGAARD, 2013a, p. 1667) che, alla sua volta, in essendo Dio, può soltanto esser trovata nello stesso spirito umano.

La seconda interpretazione, quella estetico-dogmatica, si fonda nella mozione di un senso diverso della espressione porre se stesso. Quindi, "[...] se con l'espressione 'porre qualcosa' si intende o creazione (in senso causale) o l'essere fondamento (in senso non causale) di un'essenza eterna, è escluso che il sé finito possa aver 'posto se stesso'" (LÜBCKE, 2008, p. 80). Ma se con l'espressione non si vuole sostenere quello senso anzi un altro, di carattere normativo e teleologico, dunque Lübcke (2008) pensa di essere possibile parlare di un essere finito che ponga a se stesso nella esistenza. L'alternativa da lui proposta si concentra non "[...] su ciò che il sé è o può essere (in breve tutti i tratti del sé che possono essere afferrati grazie a una descrizione) [ma invece] su ciò che il sé dovrebbe essere (vale a dire la dimensione normativa del sé)" (LÜBCKE, 2008, p. 82).

Il cambiamento di enfasi è facile di esser descritto come lo spostamento dall'ontologia alla normatività, dalla descrizione del sé alla descrizione degli scopi delle azioni del sé per mezzo delle qualle il sé diventa realtà nella esistenza. In un senso relativista di questa interpretazione normativa il primo fondamento dello scopo delle azioni umane transcorre dalle espressioni come trovo che ..., sento che... voglio che ... cioè:

[...] se ha ragione il relativista, è naturale dire che fin fine è il sé che, in modo esplicito o implicito, presup-pone determinate norme fondamentali e in questo senso normativo ha 'posto se stesso', facendosi esso stesso fondamento dello scopo delle sue azioni, o in modo sussidiario della sua vita. (LÜBCKE, 2008, p. 82). 
Questo porre le norme delle azioni per cui l'uomo diventa persona, spirito, sta in intima conessione con uno dei miei principali argomenti in questo testo: l'argomento della natura ricorsiva del processo dell'attuarsi. Tuttavia ci sembra che il argomento relativista di Lübcke non soddisfaccia tutte le possibilità che la teoria kierkegaardiana delle molteplici forme di disperazione implica o esige, molteplicità palese nello stesso concetto kierkegaardiano disperazione (Fortvivlelse), come lo spiega Hannay (2008, p. 329). Neanche la versione assolutista della interpretazione normativa ${ }^{5}$ ci sembra bastare. La versione dogmatica rifiuta il sé come quello che può decidere qualle azioni sono giuste o sbagliate, buone o cative. Però è ancora una interpretazione diversa da quella prima interpretazione teologica, anche se la conclusione alla quale giunge sia la medesima ovvero che il sé non pone a se stesso.

Una interpretazione più comprensibile deve partire dalle azioni del sé, come fa l'interpretazione normativa, ma deve anche raggiungere l'uomo nella sua natura ontologica; deve mettere insieme il se stesso e l'altro - sia quell'altro il Dio, la misura dell'uomo nella seconda parte del saggio sulla Malattia Mortale, como afferma Hannay (2008, p. 341), che gli altri uomini - nel processo dell'attuarsi dell' ' $o$. Lo stesso senso è anticipatto per Almeida (2009, p. 51-52) sotto l'idea di un "[...] drama esistenziale di diventare se stesso come una relazione derivatta, dal momento che si deve diventare se stesso però partendo da un altro". Insomma dev'essere conforme alla paradossalità della filosofia di Kierkegaard quando lui discute l'esistenza e l'uomo nell'esistenza.

Per sviluppare questa interpretazione alternativa dobbiamo tornare al senso kierkegaardiano del rapportarsi. Dunque Grøn prende di Kierkegaard lo stesso problema discusso dal Lübcke: l'uomo come un rapporto. Però in lui la questione è un po' diversa, come si può notare nell'inizio del suo testo: “[...] in Soren Kierkegaard 'l'essere umano come rapporto' ha un duplice significato. In primo luogo l'uomo è esso stesso un rapporto, in secondo luogo è un rapporto a qualcos'altro da se stesso" (GRØN, 2008, p. 103). Grøn arriverà al problema del porre a se stesso o esser posto da un altro per una via diversa da quella percorsa da Lübcke, ma il problema sarà lo stesso e questo non è altro che la ricerca della origine dell' $I o$, ossia delle condizioni per cui l'uomo diventa spirito o un Io nella esistenza, ciò che è la ricerca del rapportarsi.

Dunque il rapportarsi è il modo per cui l'uomo determina a se stesso in quanto uomo, in quanto Io. Il rapportarsi non è ciò che vai oltre l'uomo;

\footnotetext{
${ }^{5} \mathrm{La}$ versione assolutista sostiene che ci sia una fondazione ultima per le norme fondamentali e conseguentemente che alcuni scopi siano migliori di altri.
} 
non è una qualità di più ${ }^{6}$. Anzi è il percorso esistenziale per l'aggiornamento dell'uomo come una sintesi tra l'anima e il corpo verso lo spirito, fino che l'Io non possa che esser descrito come un essere composto, sintese non necessaria (prodotto della libertà) fra delle qualità o grandezze eterogenee (GRØN, 2008, p. 104). Perché sintesi non necessaria? Perché questa sintesi (come scopo) e così il rapportarsi (come il processo dell'attuarsi dell' Io) si sviluppa a partire delle scelte fatte per lo stesso uomo, ossia delle scelte fatte in libertà. Quindi Grøn (2008, p. 104) può concludere: "Possiamo formulare la domanda in questi termini: che cosa significa che l'uomo in quanto sé è una sintesi? La risposta breve è che a un uomo è posto il compito di metter in equilibrio l'eterogeneo che esso stesso è".

Ecco il fondamento della disperazione nelle sue molteplici forme (KIERKEGAARD, 2013a, p. 1689-1755). E perché l'Io esiste solo come la sintesi e l'equilibrio della diversità, a lui tocca il compito di lavorare sul se stesso, vale a dire compiere lo sforzo costitutivo di se stesso, ciò che è anche l'interiorizzazione come la costituzione di una soggettività coerente rispetto al mondo delle cose e delle altre persone (KIERKEGAARD, 1987, p. 206; 292; 304). Insomma possiamo dire che gli stadi esistenziali kierkegaardiani principalmente l'estetico, l'etico, il religioso - sono dei diversi modi per cui l'individuo risponde alle domande dell'eterogeneo in sé. E questo l'individuo fa rapportandosi a se stesso: "Rapportandosi a se stesso come anima e a se stesso come corpo, a se stesso come temporale e a se stesso come eterno", afferma Grøn (2008, p. 104).

Quello che si conclude di tutto questo è che l'essere dell'uomo, l'Io, è un essere storico e così mai è più che un progetto, una possibilità, un divenire o attuarsi, mentre la sua identità "[...] con se stesso è fragile e vulnerabile" (GRØN, 2008, p. 105). Dunque l'essenza dell'uomo, se posso così dire, è un'essenza ad attuarsi, a divenire nella esistenza. Perciò troviamo quello che Fabro (2010, p. 72-73) chiama di “[...] priorità dell'esistenza sull'essenza: l'essere dell'uomo è quello che egli diventa per la dialettica negativizzante della libertà". Ma un'altra volta Grøn (2008, p. 107) torna al punto centrale del processo di diventare l'Io: il rapporto. E allora lo sviluppa un po' più mentre discute un'altra nozione di soggettività:

\footnotetext{
${ }^{6}$ In quanto un processo, Grøn c'è ragione. Però in quanto scopo, il rapportarsi dell'uomo può prendere come obiettivo esattamente il trascendere all'uomo stesso, trascendere se stesso verso al divino, che è lo scopo dell'attuarsi nel religioso kierkegaardiano. Quindi il rapportarsi, come processo è seccuramente immanente, ma come scopo può - a dipendere dello stadio esistenziale - trascendere all'uomo stesso, e così posso dire che siamo arrivati ad un altro paradosso kierkegaardiano.
} 


\begin{abstract}
Qui ci stiamo indirizzando verso un'ermeneutica della soggettività che può attingere da idee centrali in Kierkegaard. La domanda sulla comprensione gioca un ruolo più pervasivo e più decisivo in Kierkegaard di quanto non appaia dalla letteratura su di lui. Ma l'importante è porre la domanda sulla comprensione nel suo contesto: è una domanda su cosa voglia dire un sé, ma è al contempo una domanda sul comprendere l'altro come un altro. Con questa questione della comprensione di sé - comprendere se stessi nel comprendere altri - ci troviamo nell'ambito compreso tra teoria della soggettività ed ermeneutica. (GRØN, 2008, p. 107).
\end{abstract}

E Grøn (2008, p. 107) conclude: "Il fatto che un uomo è un rapporto si può dunque intendere così: che è un essere relazionale. [...] È rapporto a sé, e lo è in rapporto ad altri”. Quindi ci troviamo allora ad un punto fondamentale che nel contempo dev'essere meglio spiegato. Così del se stesso è detto che comprenda se stesso nel comprendere l'altro e conseguentemente nel rapportasi a sé quando e perché si rapporta agli altri. Il carattere relazionale dell'Io, secondo Grøn, comprende dei due punti che sono fondamentale al mio argomento ossia che questo rapportarsi a se stesso, nel pensiero di Kierkegaard, è tanto ricorsivo nella relazione con l'altro quanto comprende un'altro che non sia soltanto il divino, ma pure l'altro umano e così impliche il lavoro di sé. Ci si può capire nello stesso testo di Grøn (2008, p. 108-109), dal momento che l'autore afferma chiaramente dei due punti quando scrive sull'interiorità: "L'interiorità diviene una questione sul comprendere se stessi in ciò che si disse e si fa in rapporto all'altro di sé"; e afferma anche la pluralità della nozione dell'altro quando usa la parola nel plurale. Però l'importanza dei due punti richiede di noi l'approfondimento dell'argomento.

Prima di tutto rischiamo a sbagliarci se ci dimentichiamo che l'interiorità o la soggettività ${ }^{7}$ nella filosofia di Kierkegaard non è soltanto un comprendere cognitivo o intellettualista ma é un vero atteggiamento che comprende principalmente la sfera pratica ovvero l'esistenza come attualità. Come scrive Fabro (2004, p. 235) "[...] sarebbe allora come se Kierkegaard al primato della sfera speculativa avesse sostituito il primato della sfera pratica, all'asse contemplativo quello attivistico, alla parola il gesto, alla dottrina l'azione". Scrive ancora in altro brano: "L'auto coscienza autentica non è contemplante sapere di sé, ma l'agire nel quale io divengo" (FABRO, 2004, p. 190).

\footnotetext{
${ }^{7}$ È principalmente nel testo della Postilla Conclusiva che Kierkegaard presenta il concetto di soggettività come azioni, ma azioni dell'interiorità (KIERKEGAARD, 1992, p. 203, 243, 339).
} 
Perciò l'Io non si aggiorna nella contemplazione di sé o nella contemplazione dell'altro, anzi lo fa in ciò che dice e fa concrettamente all'altro ovvero nell'atteggiamento e nelle azioni che prende verso alle altre persone. Il processo è relazionale e concreto invece di astratto. Tanto Grøn quanto Fabro ci fanno ricordare che la relazione dell' Io con le altre persone è atteggiamento e concretudine: è attività, gesto, azione, è dire ed è fare in rapporto all'altro come un altro.

Cosa vuol dire il carattere ricorsivo del rapporto a se stesso in rapporto (o attraverso il rapporto) ad altri? Mentre rispondo a questa domanda penso trovare dei mezzi teorici per rispondere ad un'altra domanda e per sviluppare un punto teorico centrale per il mio argomento: che l'attuarsi dell' $T o$ è un simultaneo esser posto da un altro e un porre a se stesso, entrambi i processi insieme nel rapportarsi a se stesso che, a sua volta, esige il movimento verso agli altri come altri.

Dunque l'interiorità dell'uomo, il suo $I o$, diviene nell'esistenza mentre lui comprende e sviluppa a se stesso - attuasi - in tutto ciò che dice e fa agli altri. L'altro come il divino può soltanto esser un altro come altro, giacché il punto fondamentale nei testi del solitario danese (per esempio in KIERKEGAARD, 2013b, p. 607-612) è che Dio è assolutamente diverso degli uomini. Questa diversità di qualità che non può esser sorpassata per gli uomini da soli, senza il maestro divino (KIERKEGAARD, 2013b, p. 607), è un vero indizio della realtà dell'Altro ossia il Dio, e così degli altri ossia gli uomini, nel pensiero kierkegaardiano.

Allo stesso tempo il sé si rapporta agli altri come se stesso, come scrive Grøn quando analizza quello che lui chiama un doppio rapporto, il rapportarsi a se stesso mentre si rapporta ad un altro: "È in quanto sé che esso si rapporta agli altri” (GRØN, 2008, p. 109). Così le due dimensioni del rapporto, il sé e l'altro, sono reali e efetivamente implicati nel processo. Quei del rapportarsi non sono né un sé astratto né un altro soltanto concettuale, né un sé che si riduca all'altro né un altro che si riduca al sé. Entrambi le persone del rapporto devono essere realtá in loro stessi, singolarità riconoscibili. Insomma: "[...] un uomo è esso stesso (come rapporto a sé) in rapporto ad altro da se stesso. Lo si vede nel fatto che la comprensione di sé è in gioco nel comprendere l'altro come un altro da sé” (GRØN, 2008, p. 111).

È per questa ragione che per Kierkegaard il rapporto con Dio - l'Altro per eccellenza - è il primo e più importante rapporto perchè è il fondamento 
di tutta la realtà (KIERKEGAARD, 2013a, p. 1835; FABRO, 2004, p. 262). Ma questo non cancella la possibilitá degli altri umani come gli altri con cui si rapporta il sé. Invece questo è abbastanza chiaro nel testo sugli Atti dell'Amore, dove Kierkegaard discute l'ordine o pure la raccomandazione di amare l'altro mentre il solitario danese interpreta la parabola del buon samaritano. E pure Grøn afferma: “[...] nel rapporto a Dio un uomo è rinviato all'altro uomo come prossimo" (GRØN, 2008, p. 109). Insomma lo stesso Grøn afferma il carattere paradossale del rapportarsi:

L'interpretazione che ho schizzato non ha solo un suporto testuale. $\grave{E}$ anche affascinante dal punto di vista sistemático. L'idea è che un uomo è un rapporto a sé, e che lo è in rapporto all'altro da se stesso, in rapporto al quale compreende se stesso. Ciò significa a sua volta che il rapporto a sé è avviluppato nel rapporto ad altri. Nel rapportarsi ad altri un uomo è posizionato con se stesso: con ciò che fa in rapporto agli altri posiziona se stesso - in rapporto ad altri. Dunque la domanda può essere se nel comprendere gli altri compreenda se stesso. (GRØN, 2008, p. 110).

Il paradosso si trova nel fatto che il sé e l'altro, anche se sono diversi tra di loro e diversi nei suoi fondamenti ontologici, diventano il sé e l'altro soltanto nella relazione fra di loro ${ }^{8}$. Se parliamo dell'esistenza, non ci sono un sé ed un altro-di-sé fuori della relazione fra il sé e l'altro. Per questo è che abbiamo visto anzitutto che il sé è sempre relazionale. Più di questo posso affermare che l'Io si sviluppi come un sé non soltanto nella relazione con l'altro, ma pure che il sé solo possa rapportarsi ad altri come altri quando è già soggetto, personalità, un $I o$ diverso e riconoscibile in diversità rispetto agli altri. Se l'Io è ancora una indistinzione allora non ha un sé per rapportarsi all'altro. Ci siamo arrivati alla paradossalità nel cuore del processo dell'attuarsi come persona, come l'Io nell'esistenza: il sé esiste in dipendenza all'altro però solo riesce a rapportarsi ad altri quando è già un sé identificabile o che si venga progettato indietro verso se stesso (GRØN, 2008, p. 114).

Concludo pertanto che l'altro - sia come il Dio che come gli altri uomini - non è soltanto un dettaglio, una minutezza. Anzi è il fulcro del processo del rapportarsi a se stesso per cui l'uomo diventa l' $I o$ : “[...] un uomo è un sé nel rapportarsi ad altro da sé. [...] un uomo è un sé di fronte a, è esso stesso in rapporto a" (GRØN, 2008, p 109). Il sé e l'altro vanno insieme nel

\footnotetext{
${ }^{8}$ Almeno quando l'altro di chi si parla è un altro essere umano, e non Iddio che, ovviamente e nel senso preso dal solitario danese - ci si veda per esempio la nozione del divino nelle Briciole di Filosofia non può esser posto dalla relazione con le persone umane.
} 
processo, senza che nessuno possa dire dove cominci o finisca quel processo e senza che uno venga all'esistenza senza l'altro.

Ho stabilito che il porre a se stesso ovvero il lavoro di sé, nella filosofia di Kierkegaard, esige la dimensione relazionale del rapportarsi agli altri. In una tale relazione l'uomo non attua se stesso in corrrespondenza alla natura - nella qualle l'uomo non può che essere soltanto ripetizione e indistinzione rispetto alla specie - ma in corrispondenza alla sua più sublime possibilità: la coscienza del sé come unico (unità con se stesso) e perciò il riconoscimento ed il rispetto dell'altro pure come unico, mentre l'uno e l'altro sono simili fra di loro e non sono inferiori verso alla colletività. Quello che spero discutere adesso è che questo senso di rapporto e anche la nozione di un altro come vero altro si può trovare soltanto nel religioso, dove l'altro diventa il prossimo, il primo-Tu.

\section{L'UOMO È UN RAPPORTARSI, MA A CHI SI RAPPORTA L'UOMO? L'ALTRO NEGLI STADI ESISTENZIALI}

La nozione kierkegaardiana di stadio esistenziale appunta verso le diverse possibilità o modo di esistenza, i diversi percorsi per cui l'uomo costituisce la sua storia personale e singolare nel tempo per mezzo delle scelte che lui stesso fa in ogni istante della sua esistenza. La discussione qui tocca del processo per cui l'essere umano diventa soggettività integra con sé stessa, ciò che si può raggiungere per mezzo di tre principali percorsi.

Caso si voglia parlare di questi percorsi come modi di rapporto, allora possiamo dire che nell'estetica l'essere umano è un rapporto che si rapporta alla natura in se stesso, vale a dire alle forze e spinte naturali che operano nell'intimo dell'individuo umano. In questo senso l'individuo nell'estetico è soltanto un rappresentante della spezie umana, indifferenziato rispetto a qualunque altro individuo e che perciò non diventa propriamente personalità singolare (KIERKEGAARD, 1987, p. 159).

Come accade agli altri animali, il percorso estetico porta l'uomo verso lo stadio della indistinzione in cui l'interiorità resta una ripetizione senza movimento (KIERKEGAARD, 1987, p. 178), senza attuarsi - davvero senza libertà, almeno in quanto la libertà si traduca come impegno verso una soggettività coesa (FABRO, 2013, p. 65) che è l'individualità personale o spirito (FABRO, 2010, p. 65). Tuttavia è giusto parlare dell'estetica come un percorso in direzione dell' $I o$ ? Ci sembra possibile una risposta affermativa, poichè anche 
l'estetico esige una decisione fondante del sé: quella di non impegnarsi anzi lasciarsi al momento ed alle tutte le possibilità che il tempo gli traga. Dunque dal punto di vista della scelta di sé anche l'estetico è responsabile per se stesso: egli sceglie di non scegliere a se stesso. E quindi anche l'estetico si può tradurre come un percorso esistenziale legittimo, percorso tuttavia che porta l'uomo soltanto a quello che in lui stesso è finito e che, nell'ambito dello spirito (della coscienza) é molteplicità con sé ed in se stesso.

Insomma l'estetico in Kierkegaard si traduce come la mancanza di senso dell'esistenza per chi non riesce a vederla come più che una semplice raccolta d'innumerevoli momenti, ognuno di cui vuoto di senso nello stesso modo che l'esistenza in generale diventa ugualmente vuota di senso. Davanti all'esistenza vuota l'esteta si dispera e fa la sua unica scelta esistenziale e costitutiva di sé: sceglie lasciarsi alle possibilità e persegue ogni opportunità che l'esistenza gli offra ma soltanto fino al limite del suo godere immediato. Perciò dell'esteta si può dire che lui sia un edonista però è ancora meglio che si dica quello che lui non raggiunge: come il prodotto dell'occasione lui non diventa un Io coeso o spirito nella esistenza. Come si legge nel Diario del Seduttore: "Prima tutto stava nel fatto che in modo egoisticamente personale godesse sia di quello che la realtà gli dava, sia di quel con cui aveva fecondato la realtà; poi la sua personalità veniva volatilizzata, ed egli allora godeva della situazione e di se stesso nella situazione" (KIERKEGAARD, 2012, p. 46).

Questo è l'esteta kierkegaardiano rispetto a se stesso. Rispetto all'altro ci troviamo in condizione non molto diversa, giacché l'esteta si rapporta al mondo sulla base del suo sfruttamento (KIERKEGAARD, 1988, p. 72), che si traduce come la spinta per approfittare ogni opportunità in modo indifferente rispetto a se stesso. Quindi l'altro diventa questa opportunità, l'occasione per la fruizione immediata o il godimento di se stesso (o della natura in se stesso) nell'altro, per cui lo scrittore del Diario del Seduttore potrà affermare: "Gli individui non sono stati per lui che uno stimolo" (KIERKEGAARD, 2012, p. 49). E anche:

Sarà dunque il principio strategico, la legge per tutti i movimenti [...] Ecco perché le mie storie d'amore hanno sempre una realtà per me stesso. [...] e spesso vi si associa perfino qualche dote pratica: imparai a danzare a causa della prima fanciulla che amai, imparai a parlar francese a causa d'una ballerinetta. (KIERKEGAARD, 2012, p. 97). 
Concludiamo pertanto che l'altro nell'estetico è l'occasione per sfrutare la esistenza in tutto quello che ella offre. Un vero altro non c'è, e forse nemeno un vero $I o$, almeno un $I o$ coerente o coeso in se stesso oltre la dissoluzione, la molteplicità interiore secondo la molteplicità delle oportunità che la esistenza gli offra.

Già l'etico consiste in attuarsi come un Io coerente in se stesso. Contro la molteplicità dell'Io estetico, l'etico è lo stadio dell'integrazione del sé con se stesso per mezzo delle azioni interior' (KIERKEGAARD, 2009, p. 131). Nell'etico il rapporto a sé è anche o fondamentalmente scelta di se stesso in ogni momento dell'esistenza. Nell'etico si dice che l'umano è un rapporto che si rapporta a se stesso ed è anche il prodotto del lavoro di porre a se stesso. L'etico è l'esercizio della volontà sopra la natura - la natura nello stesso uomo. Ed è anche il processo di porre a se stesso per mezzo del rapporto con l'altro perchè - come ne abbiamo già visto - l'etico fa la distinzione fondamentale tra l'essenziale e l'accidentale nella esistenza (KIERKEGAARD, 2009, p. 153) per diventare se stesso. Quindi l'etico deve rapportarsi all'altro per distinguere a se stesso e per attuarsi in modo coerente nella esistenza. Però quel rapporto all'altro non c'è l'altro come punto di riferimento, anzi c'è l'Io stesso come il riferimento e così è ancora un lavoro di auto-riferimento e di auto-aggiornamento. Si nella sua missione, nel suo impegno volitivo per diventare se stesso nell'esistenza, l'etico mette l'uomo in contato con gli altri uomini (KIERKEGAARD, 2009, p. 212) il suo dovere è comunque dovere verso se stesso: "L'individuo etico non ha insomma il dovere al di fuori di sé" (KIERKEGAARD, 2009, p. 149).

L'etica in Kierkegaard non é comunque repetizione ma invece elezione. Benchè si possa dire che sia repetizione nella coscienza dell'uomo, non è certamente repetizione nell'esistenza. L'esistenza è per l'etica o anche per l'etico, un dovere - dovere di essere se stesso, dovere verso se stesso come una personalità integra in mezzo alla frammentarietà delle possibilità esistenziale. Così l'etica consiste di rapporto ad un Io ideale che diventa poi esistente ossia reale nella esistenza, per mezzo del lavoro di sé e dell'attuarsi in riferimento a se stesso: "L'individuo ha la sua teleologia in se stesso, [...] è a se stesso la sua teleologia; il suo sé è dunque la meta, il fine verso cui s’indirizza il suo sforzo

${ }^{9}$ C'è, in Kierkegaard - nella parte della sua opera che si può chiamare la produzione etica - una chiara distinzione tra l'etico come uno stadio esistenziale e come comprenzione dell'esistenza. In questo secondo senso l'etica è una scienza ideale, come scrive Kierkegaard nell'introduzione del Concetto di Angoscia (KIERKEGAARD, 1980, p. 16-17), ma non è questo il senso a cui mi ripporto qua anzi al primo senso ossia dell'etica come modo di esistere. 
[...]; In tal modo il movimento di colui diventa dunque a partire da se stesso verso se stesso attraverso il mondo" (KIERKEGAARD, 2009, p. 172).

Siccome l'etico prende se $\operatorname{stesso}^{10}$ (un universale dell' Io o di sé) come il suo punto di riferimento, allora l'etico può soltanto arrivare a se stesso ${ }^{11}$ e così il mondo - e le altre persone, l'altro in qualunque senso - diventa solo uno strumento, però in senso diverso dal senso estetico. Almeno qua, nell'etico, si può dire che ci sia movimento dal sé verso se stesso, movimento per cui, come scrive il solitario danese, il mondo non è più di un mezzo. Almeno nel ruolo di mediazione l'altro, nell'etico, è la condizione perchè l'Io diventi se stesso, e così si può dire che l'altro sia una proiezione dello stesso sé che nell'esistenza si attua. Non è ancora un altro con realtà diversa da quella del sé, per lo meno dal punto di vista del sé. Come scrive Kierkegaard nel testo degli Atti dell'amore (KIERKEGAARD, 2003, p. 277) il primo Io trova qua un'altro $I o$, "[...] ossia il primo Io un'altra volta, ad un livello superiore", ma che non è ancora l'altro come un altro. $\mathrm{O}$ ancora per ne parlare di un altro modo, l'altro non si fa uguale all' Io perchè, nel processo di aggiornamento del sé nell'etico, l'altro è soltanto un punto di passaggio, un negativo del sé nel suo percorso verso se stesso, un secondo Io.

Questo punto di riferimento è proprio quello che cambia nel cambiamento dello stadio etico rispetto allo stadio religioso (quando si sceglie il religioso, giacché dall'etico al religioso non si può trovare nessuna linea o successione necessaria ${ }^{12}$ ). Nel religioso l'uomo raggiunge la realtà quando sceglie Dio come riferimento del suo attuarsi e quindi il telos della esistenza non è più l'Io stesso ma trascende l'Io: il divino diventa il suo scopo esistenziale ovvero la meta del suo attuarsi (KIERKEGAARD, 2013a, 1813-1814) e così questo attuarsi, come un lavoro del sé $^{13}$, non può più esser recato ad effetto per l'uomo da solo.

\footnotetext{
${ }^{10} \mathrm{Al}$ meno l'etico nel senso compreso nel testo sopracitatto ossia del testo dell'Enten-Eller, dato che in altri testi, sotto altri pseudonimi, come nella Postilla Conclusiva (KIERKEGAARD, 1992), l'etico prende a volta aspetti diversi (ciò che non discutto qua per non uscire fuori dal mio argomento centrale in questo testo).

${ }^{11}$ Come ne ha percepito il Fabro (2010, p. 96) in rapporto con il trascendente l'etico non si trova in migliore situazione dell'estetico: "Se al di sopra dell'uomo non c'è Dio che fondi appunto lo ec-sistere dell'uomo come garanzia, sostegno e termine effettivo del medesimo, l'esistenza si esaurisce in se stessa, come inutile e doloroso contorcimento di sé in sé".

${ }^{12}$ Infatti non ha nessuna linea o progressione o anche percorso necessario nella teoria kierkegaardiana degli stadi. Qua ci serve la nozione di salto per ben capire il passaggio di un stadio all'altro, perché tutti i tre stadi sono possibilità in ogni momento, in ogni istanti della vita di ogni persona.

${ }^{13}$ Adesso un attuarsi verso Dio, verso il paradosso (o scandalo) del Dio-uomo, "la determinazione più decisiva possibile della soggettività" (KIERKEGAARD, 2013a, p. 1825).
} 
Senza dubbio questo telos, nel religioso, è un principio paradossale perché pone all'uomo il lavoro di diventare un sé - l'Io - oltre se stesso. Comunque nel religioso (e questo punto è centrale nelle Briciole di Filosofia) l'umano non è lasciato da solo nel suo lavoro, nel suo attuarsi (KIERKEGAARD, 2013b, p. 619-637 $\left.{ }^{14}\right)$. Ed allo stesso tempo si deve sapere che il rapporto a Dio è, nel religioso kierkegaardiano, rapporto anche con se stesso per diventare soggetto (GIORDANO, 2011, p. 75; PIAZZESI, 2009, p. 145), un sé che deve acquistare una nuova qualità, l'eternità o anche la felicità eterna che solo il dio-nella esistenza può portargli. E questa felicità eterna è, per dire di un altro modo, la propria realtà possibile all'uomo dato che quando lui sceglie Dio come riferimento del suo attuarsi questa scelta diventa determinante di una nuova qualità esistenziale, quella del paradosso dell'uomo-Dio.

Questo è il punto ontologico preso da Cornelio Fabro (2004) per spiegare il religioso in Kierkegaard, vale a dire, che Dio in essendo la base di tutta la realtà può essere - e soltanto Dio può essere - la fonte unica del vero cambiamento dell'esistenza umana verso la sua più alta vocazione e la sua più sublime possibilità: Dio stesso. Questa religiosità per Kierkegaard è prima di tutto comunicazione esistenziale o azione (KIERKEGAARD, 1992, p. 431). Dunque quella nuova qualità non è una idea ma un modo di vita, di esistenza (KIERKEGAARD, 1992, p. 570-571) che però deve trarre l'eterno alla esistenza, nel modo che il rapporto con se stesso si deva fare per mezzo del rapporto con l'Altro, cioè Dio. Perciò nel religioso il lavoro di sé - l'attuarsi - non è più un sforzo immanente, non comincia e finisce nel se stesso e per cui l'altro è soltanto un mezzo, un intermedio, una semplice immagine del sé o, un altro Io. Propriamente perché quello che l'uomo può e deve diventare nel religioso (la sua determinazione più decisiva possibile) viene non da sé ma dall'Altro è che in questa condizione l'uomo diventa nel modo più evidente un'eterogeneità (GRØN, 2008, p. 104). Questo si può leggere anche nel trattato sulla Malattia Mortale:

La potenziazione nella coscienza dell'io è qui la conoscenza di Cristo; l'io si trova di fronte a Cristo. [...] si è parlato dell'ignoranza di avere un io eterno; poi della coscienza di avere un io nel quale c'è qualcosa di eterno. Poi [...] si è dimostrata la differenza contenuta in questa coscienza: l'io che ha di se stesso un'idea umana o la cui misura è l'uomo, in contrasto all'io di fronte a Dio, [...]. Un io di fronte a Cristo è un io potenziato da un'immensa concessione di Dio $[. .$.$] più idea di Cristo più io. Un io è qualitativamente ciò ch'è la sua$

${ }^{14}$ E possiamo leggere anche nel testo della Postilla: Paradoxically-religiously, the teacher is the god [Guden] in time, the pupil is a new creation (KIERKEGAARD, 1992, p. 573). 
misura[...] in Cristo è vero che Dio è meta e misura, ovvero misura e meta dell'uomo. (KIERKEGAARD, 2013a, p. 1813).

Chi è l'altro nell'ambito del religioso che il proprio Dio! E pertanto è un altro che è allo stesso tempo assoluto e assolutamente diverso dall'uomo, nel senso ontologico (KIERKEGAARD, 2013a, p. $1825^{15}$; KIERKEGAARD, 2013c, p. $1925^{16}$ ). A questa costatazione si relaziona pure l'affermazione che si legge nella parte conclusiva del testo di Enten-Eller, il cosiddetto Ultimatum: “di fronte a Te abbiamo sempre torto" (KIERKEGAARD, 2009, p. 257). Pertanto nel religioso tutti gli uomini sono uguali ma soltanto perché, di fronte a Dio, tutti sono torti $^{17}$, per cui possiamo sicuramente sapere che in questo stadio Kierkegaard fissa una distinzione profonda e definitiva tra l'uomo e Dio, tra l'Io, il sé nell'esistenza e l'altro, quando quest'altro è Dio ovvero l'assoluto (KIERKEGAARD, 2009, p. 271).

La distinzione prende il suo senso dal fatto che nei rapporti verso Dio l'altro del sé umano è un altro assolutamente - cioè qualitativamente - diverso dell'uomo, come ne abbiamo già vista ${ }^{18}$. È pertanto e per questo che nello stadio religioso l'altro prende valore in sé innanzi all' $I o$. Però nel religioso l'altro è tanto quel maestro divino che porta l'Io oltre se stesso - verso il divino - quanto gli altri uomini che devono esser amati dall'Io perché è questo il comandamento divino, "[...] tu devi amare 'il prossimo" (KIERKEGAARD, 2003, p. 245 e p. 269) e perché loro sono ontologicamente uguali (KIERKEGAARD, 2003, p. 279). Dio - il primo Altro - non può che esser trovato dallo stesso Io che nel rapporto all'altro sotto la condizione del vero amore.

L'altro umano diventa così il prossimo in relazione all'Io e pure l'Io diventa il prossimo in relazione alle altre persone, sulla base del comandamento dell'amore e sul fondamento della loro uguaglianza ontologica. Come scrive Kierkegaard, si

[...] l'amore spirituale invece elimina dal mio Io ogni qualità naturale ed ogni egoismo, perciò l'amore del prossimo non può trasformarsi in una cosa sola col prossimo in un Io unificato. L'amore del prossimo è l'amore fra due nature

15 "Come peccatore l'uomo è separato da Dio dalla profondità più abissale della qualità".

16 “C'è infatti una differenza abissale infinita fra Dio e l'uomo".

${ }^{17}$ Importa qui riferire l'interessante discussione che Valls (2000, p. 188-123) conduce sulla definizione dell'amore cristiano come dovere di amare il diverso, il differente, il dovere di amare l'altro proprio perché l'altro é diverso dall'Io.

${ }^{18}$ Allora nel senso religioso il rapporto fra l'uomo e Dio non può esser giudicato che come un paradosso. 
determinate in eterno ciascuna per sé come spirito [...] due spiriti non possono mai diventare un solo Sé [...] (KIERKEGAARD, 2003, p. 275).

Concludiamo allora che nel religioso l'altro prende il suo senso più forte nella filosofia kierkegaardiana dal momento ch'è definito come Dio ma anche, nel senso umano, come il prossimo, il mio somigliante, il primo Tu: "Sia che parliamo del primo Io sia del secondo, non abbiamo fatto un passo per giungere al prossimo, poiché il prossimo è il primo Tu” (KIERKEGAARD, 2003, p. 277).

\section{Conclusione}

Ciò che ne abbiamo visto in questa discussione può esser riassunto con le tre espressioni che io ho preso per parlare della nozione dell'altro negli stadi esistenziali: mentre nell'estetico l'altro è occasione e nell'etico è un altro Io, il secondo Io, nello stadio religioso l'altro é Dio ed è anche gli altri uomini, il primo Tu. Se il nostro è un tentativo per stabilire alcuni punti fondamentali per una filosofia sociale kierkegaardiana, dunque si vede che il religioso dev'essere nostro punto focale.

L'amore del prossimo è il punto fondamentale del discorso sugli Atti dell'Amore ossia è il comandamento per cui, secondo lo stesso Kierkegaard (2003, p. 245) l'uomo “[...] scopre e sa che il prossimo esiste e [...] che ognuno lo è. Se non ci fosse il precetto di amare, non esisterebbe neppure il concetto di prossimo; ma soltanto quando si ama il possimo, allora soltanto è sradicato l'egoismo della preferenza ed è conservata l'uguaglianza dell'eterno". Eccolo qua, secondo il proprio Kierkegaard, il percorso dall'Altro divino all'altro umano, giacché quel rapporto verso l'altro c'è Dio - il primo Altro - come il suo autore, come la condizione intermedia del rapporto fra l'uomo e l'uomo (KIERKEGAARD, 2003, p. 279) e quando si ama l'altro, si arriva allo stesso Dio, per mezzo degli atti del vero amore ${ }^{19}$ (FABRO, 2003, p. 112), vale a dire, in quello che si fa e si dice all'altro, come ne abbiamo già visto.

Dunque nel religioso il lavoro di sé, l'attuarsi dell'uomo può esser spiegato come un attuarsi del sé verso ad una possibilità che si trova oltre se stesso, una possibilità che Kierkegaard designa come coscienza eterna, beatitudine

\footnotetext{
${ }^{19}$ La distinzione ed anche la relazione dialettica tra l'amore profano (Elskov) e l'amore sacro (Kjerlighed), fondamentale per capire il rapportarsi (anche questo) dialettico fra l'uomo-Dio-il prossimo può esser trovata nel saggio di Fabro (2003, p. 83-104).
} 
eterna (KIERKEGAARD, 2013b, p. 591), l'eternità o realtà nel suo significato essenziale. L'uomo diventa se stesso, nel religioso, insieme all'altro e questo può esser un importante principio per una filosofia sociale kierkegaardiana, sulla base della nozione di dono, dono di sé all'altro e dono che l'altro fa all' Io perchè questo diventi l'uomo in sua più nobile possibilità: l'uomo con una vocazione ciò che si può anche chiamare una coscienza - eterna.

CRUBELLATE, Joano Marcelo. The notion of the other in Kierkegaard's theory of existential stages. Trans/Form/Ação, Marília, v. 38, n. 1, p. 89-102, Jan./Abr., 2015.

\begin{abstract}
From the notion of self as a relation's relating itself to itself, I discuss the concept of the other in relation to the self in each of the Kierkegaardian life stages: the esthetic, the ethical, and the religious. First of all, I describe the notion of self in Kierkegaardian philosophy as it appears in Sickness unto Death (Kierkegaard, 2013a), and the problem of the human being as a conscience in the world. After that, I describe the esthetic notion of the other, which is no more than a casual opportunity of the self for enjoying itself in life. In the ethical stage, the other is described as an image of the self, a second-self: no more than an intermediate between the self and the same self. Finally, the religious stage is arrived at, where the other is, for the self, another being with independent reality. There the other is, firstly, God the true source of all reality and the life of the self - and it is the biblical neighbor with whom the self must establish itself as a singular being. The religious other is then the basis of the Kierkegaardian social philosophy, since it is not a second-I but the first-Thou (KIERKEGAARD, 2003).
\end{abstract}

KEYWORDS: Kierkegaard. Relating. Stages of life. Neighbor.

\title{
REFERÊNCIAS
}

ALMEIDA, Jorge Miranda. Ética e existência em Kierkegaard e Lévinas. Vitória da Conquista: Ediçōes UESB, 2009.

DE PAULA, Marcio Gimenes. Subjetividade e objetividade em Kierkegaard. São Paulo: Annablume, 2009.

FABRO, Cornelio. Saggio Introduttivo. In: KIERKEGAARD, S. Atti Dell'Amore. Milano: Bompiani, 2003.

. Dall'essere all'esistente. Milano: Marietti, 2004.

Tra Kierkegaard e Marx. Segni: Editrice del Verbo Incarnato, 2010.

. Introduzione. In: KIERKEGAARD, Søren. Le grandi opere filosofiche e teologiche.

Milano: Bompiani, 2013. p. 21-103.

Søren Kierkegaard: il problema della fede. Segni: EDIVI, 2014. 
GIORDANO, Diego. Verità e paradosso in Søren Kierkegaard. Napoli: Orthotes, 2011.

GRØN, Arne. Comprensione di sé e dialettica della comunicazione. In: ROCCA, Ettore (Ed.). Søren Kierkegaard - L'essere umano come rapporto. Brescia: Mocelliana, 2008. p. 103-118.

HANNAY, Alastair. Kierkegaard and the variety of despair. In: ; MARINO, Gordon (Eds.). The Cambridge Companion to Kierkegaard. Cambridge: Cambridge University Press, 2008. p. 329-348.

KIERKEGAARD, Søren. The concept of anxiety. Princeton: Princeton University Press, 1980.

. Either/Or (Part II). Princeton: Princeton University Press, 1987.

. Stages on life's way. Princeton: Princeton University Press, 1988.

. Concluding unscientific postscript to philosophical fragments. Princeton: Princeton University Press, 1992. v. 1. . Atti Dell'Amore. Milano: Bompiani, 2003.

. Enten-Eller (Tomo Quinto). 3. ed. Milano: Adelphi, 2009.

. Enten-Eller (Tomo Terzo). 4. ed. Milano: Adelphi, 2012.

. La malattia mortale. Milano: Bompiani, 2013a. p. 1655-1840. (Le Grandi Opere Filosofiche e Teologiche)

. Briciole di filosofia. Milano: Bompiani, 2013b. p. 591-744. (Le Grandi Opere Filosofiche e Teologiche)

. Esercizio del cristianesimo. Milano: Bompiani, 2013c. p. 1841-2190 (Le Grandi Opere Filosofiche e Teologiche).

LÜBCKE, Poul. Aver posto se stesso o essere stato posto da un altro. In: ROCCA, E. (Ed.). Søren Kierkegaard: l'essere umano come rapporto. Brescia: Mocelliana, 2008. p. 73-88.

MORELLI, Sergio. L'emergere della soggettività in Kierkegaard. Milano: Glossa, 2012.

PIAZZESI, Chiara. La verità come trasformazione di sé. Pisa: Edizione ETS, 2009.

REGINA, Umberto. Stato, chiesa e uguaglianza di tutti gli uomini. In: ROCCA, Ettore (Ed.). Søren Kierkegaard: l'essere umano come rapporto. Brescia: Mocelliana, 2008. p. 3-14.

VALLS, Alvaro. Entre Sócrates e Cristo: ensaios sobre a ironia e o amor em Kierkegaard. Porto Alegre: EDIPUCRS, 2000.

Recebido em: 03/06/14

Aceito em: 03/09/14 
CRUBELlate, J. M. 